\title{
Quantum-information processing in strongly detuned optical cavities
}

\author{
E. Jané, ${ }^{1,2}$ M. B. Plenio, ${ }^{2}$ and D. Jonathan ${ }^{3}$ \\ ${ }^{1}$ Departament d'Estructura i Constituents de la Matèria, Universitat de Barcelona, Diagonal 647, E-08028 Barcelona, Spain \\ ${ }^{2}$ QOLS, Blackett Laboratory, Imperial College of Science, Technology and Medicine, London SW7 2BW, United Kingdom \\ ${ }^{3}$ DAMTP, Centre for Mathematical Sciences, University of Cambridge, Wilberforce Road, Cambridge CB3 OWA, United Kingdom
}

(Received 17 December 2001; published 2 May 2002)

\begin{abstract}
We demonstrate that quantum-information processing can be implemented with ions trapped in a far detuned optical cavity. For sufficiently large detuning the system becomes insensitive to cavity decay. Following recent experimental progress, this scheme can be implemented with currently or soon available technology.
\end{abstract}

DOI: 10.1103/PhysRevA.65.050302

PACS number(s): 03.67.Hk

Quantum information and quantum entanglement are the key resources in quantum-information science [1]. Achieving their controlled experimental manipulation is of paramount importance for the actual implementation of any quantum information processing protocol.Many schemes for the practical implementation of quantum-information processing have been proposed in the past. Amongst those, one of the most promising approaches towards controlled and scalable quantum-information processing employs linear ion traps. They offer the possibility in principle to store strings of ions, cool them almost to zero temperature, and address them individually with laser light [2]. The individual ions can interact with one another via their motional degrees of freedom. Indeed, it was realized early on that, based on these features, ion traps allow for the implementation of all quantum gates that are necessary for universal quantum computation [3]. In practice, however, the implementation of this proposal is less straightforward. Very low temperatures have to be achieved and subsequent heating of the ion motion as well as spontaneous emission from the ions has to be suppressed [4]. Many proposals such as heat-insensitive quantum gates [5-7] and the use of Raman transitions and interference-aided laser cooling [8] have been made to address these problems. Nevertheless, the requirement of achieving low temperatures in the motional degrees of freedom of the ions proves to be a significant problem in actual experiments.

Recently, approaches have therefore been proposed that employ ions that are trapped inside of an optical cavity. Instead of using the mechanical degrees of freedom of the ions to mediate their interaction, here the optical-cavity mode plays that role. The inevitable cavity decay through the mirrors appears to cause a problem. However, it has been realized that in fact this cavity decay may be used to create entanglement between ions in cavities [9] and to establish quantum communication networks between different optical cavities [10].

In this paper we also study a system comprising trapped ions inside an optical cavity, a system that has been demonstrated experimentally by various groups [12]. The cavity mirrors are not perfect, but as opposed to earlier proposals, we do not employ this cavity decay but rather use the idea that a strongly detuned cavity can be used to suppress cavity decay. In fact, the use of strongly detuned cavities will effectively decohere all those transition amplitudes that would lead to a population of the cavity mode, thereby suppressing the decay of the cavity. The spontaneous decay of the internal electronic states of the ions can also be reduced by using ions in a configuration where two ground states representing the qubit are coupled via far detuned Raman transitions.

In the following we will demonstrate that with this setup comprising trapped ions and a far detuned cavity we are able to implement both-single qubit rotations as well as two-qubit gates such as the (controlled-NOT) gate. Therefore, universal quantum computation can be achieved in our system. We also present a physical picture, based on the dynamical Stark shifts, to provide insight into the physical origin of the function of the gates.

Consider the situation in which there are two ions in a cavity as shown in Fig. 1. We assume that the ions are separated by at least one optical wavelength. Both ions have the internal structure of a lambda system and the optical cavity couples the states $|2\rangle$ and $|3\rangle$ of each ion. The lower levels, states $\left\{|1\rangle_{i},|2\rangle_{i}\right\}$ ( $i=1,2$ denotes the ion), form our qubit, while $|3\rangle$ is an auxiliary state. The energy level structure for one of the ions is shown in Fig. 2. The Hamilton operator describing the combined cavity-ion system can be written as

$$
\begin{aligned}
H= & \omega_{2}\left(\sigma_{22}^{1}+\sigma_{22}^{2}\right)+\omega_{3}\left(\sigma_{33}^{1}+\sigma_{33}^{2}\right)+\omega_{c} a a^{\dagger} \\
& +g\left\{a^{\dagger}\left(\sigma_{23}^{1}+\sigma_{23}^{2}\right)+\text { H.c. }\right\},
\end{aligned}
$$

where $\sigma_{\alpha \beta}^{i}=|\alpha\rangle_{i i}\langle\beta|, g$ is the cavity-ion coupling constant, $a$ and $a^{\dagger}$ are the annihilation and creation operators for the cavity photons and $\hbar=1$.

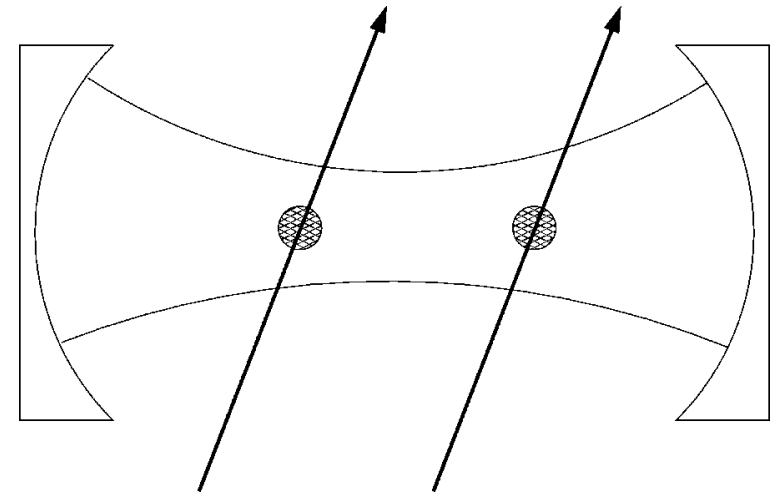

FIG. 1. We consider a setup in which two ions are trapped inside of a strongly detuned optical cavity. For our scheme to work, the ions have to be separated by at least one optical wavelength. 


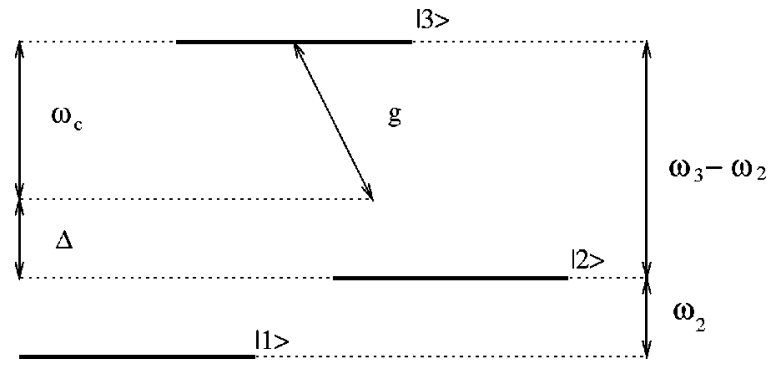

FIG. 2. Energy diagram of one of the ions. The cavity drives the transition $|2\rangle \rightarrow|3\rangle$ with coupling constant $g$ and has a detuning $\Delta$.

As mentioned in the introduction, the cavity is strongly detuned, more precisely, we assume, that the detuning $\Delta$ $=\omega_{c}-\left(\omega_{3}-\omega_{2}\right)$ and the cavity-atom coupling constant satisfies the condition $g^{2} / \Delta^{2} \ll 1$. A consequence of the strong detuning of the cavity is that the population in the cavity mode will be very small at all times if initially it is not populated. As states involving photons play a negligible role in the time evolution, we can eliminate them from the Hamilton operator adiabatically [14]. To do this we compute the effective Hamiltonian [in an interaction picture with respect to $\left.H_{0}=\Sigma_{i}\left(\omega_{2} \sigma_{22}^{i}+\omega_{3} \sigma_{33}^{i}\right)+\left(\omega_{3}-w_{2}\right) a a^{\dagger}\right]$ to second order for the subspace with zero photons, which yields

$$
\begin{aligned}
H_{\mathrm{eff}}= & -\frac{g^{2}}{\Delta}\left(\sigma_{11}^{1} \sigma_{33}^{2}+\sigma_{22}^{1} \sigma_{33}^{2}+\sigma_{33}^{1} \sigma_{11}^{2}+\sigma_{33}^{1} \sigma_{22}^{2}+2 \sigma_{33}^{1} \sigma_{33}^{2}\right. \\
& \left.+\sigma_{32}^{1} \sigma_{23}^{2}+\sigma_{23}^{1} \sigma_{32}^{2}\right)
\end{aligned}
$$

All contributions that could induce transitions to levels involving photons are rapidly oscillating due to the strong detuning of the cavity mode.

Furthermore, from Eq. (2) we see that the detuned cavity induces an energy shift in the energy levels of the atoms. The result is that the state

$$
\left|\Psi_{a}\right\rangle=\frac{1}{\sqrt{2}}(|23\rangle-|32\rangle),
$$

together with the states $\{|11\rangle,|12\rangle,|21\rangle,|22\rangle\}$ are not shifted whereas the rest are shifted by an amount of order $g^{2} / \Delta$. The goal will be to use these energy shifts to create a conditional dynamics of the two qubits.

Now we drive the transition $|2\rangle_{i} \rightarrow|3\rangle_{i}$ in both ions with two lasers with Rabi frequency $\Omega_{1}=\Omega$ for ion 1 and $\Omega_{2}=$
$-\Omega$ for ion 2 . The full Hamiltonian of the system in the interaction picture, taking into account Eq. (2), is

$$
\begin{gathered}
H^{\prime}=H_{1}+H_{2} \\
H_{1}=-\frac{g^{2}}{\Delta}\left(\sigma_{11}^{1} \sigma_{33}^{2}+\sigma_{22}^{1} \sigma_{33}^{2}+\sigma_{33}^{1} \sigma_{11}^{2}+\sigma_{33}^{1} \sigma_{22}^{2}+2 \sigma_{33}^{1} \sigma_{33}^{2}\right. \\
\left.+\sigma_{32}^{1} \sigma_{23}^{2}+\sigma_{23}^{1} \sigma_{32}^{2}\right) \\
H_{2}=\Omega\left(\sigma_{32}^{1}-\sigma_{32}^{2}+\text { H.c. }\right)
\end{gathered}
$$

If the condition $\left(g^{2} / \Delta\right)^{2} \gg \Omega^{2}$ is satisfied, we can again use perturbation theory [13] to find the effective Hamiltonian for the subspace where the states that we want to use as qubits belong to. From Eq. (4) one can see that this subspace is $\left\{|11\rangle,|12\rangle,|21\rangle,|22\rangle,\left|\Psi_{a}\right\rangle\right\}$, since the rest of the states are shifted out of resonance by the interaction of the ions with the cavity. The effective Hamiltonian to first order gives

$$
H^{\prime \prime}=\Omega \sqrt{2}\left(|22\rangle\left\langle\Psi_{a}|+| \Psi_{a}\right\rangle\langle 22|\right) .
$$

Note that because some of the levels are shifted, the laser only couples the state $|22\rangle$ with the state $\left|\Psi_{a}\right\rangle$. The resulting effective Hamiltonian is thus nonlocal despite the local interaction of the laser with the ions. The explanation for this apparent contradiction lies in the non-local character of the ion-cavity interaction. The Hamiltonian Eq. (5) can be employed to perform the c-NOT gate because the transformation

$$
\begin{gathered}
|11\rangle \rightarrow|11\rangle, \\
|12\rangle \rightarrow|12\rangle, \\
|21\rangle \rightarrow|21\rangle, \\
|22\rangle \rightarrow-|22\rangle
\end{gathered}
$$

can be obtained via $e^{-i H t}$ after a time $t=(\pi / \sqrt{2}) \Omega^{-1}$, since after a complete oscillation between the state $\left|\Psi_{a}\right\rangle$ and $|22\rangle$ the latter gains a phase, whereas the rest of the states do not evolve. This control-phase gate can be turned into a c-NOT gate by applying before and after it a Hadamard transformation.

The physical idea behind this scheme is presented in Fig. 3 where we depict the ionic energy levels corresponding to zero and one photons in the cavity mode. The off-resonant coupling between the ion and the cavity (indicated by arrows) induce Stark shifts in some of the energy levels corre-

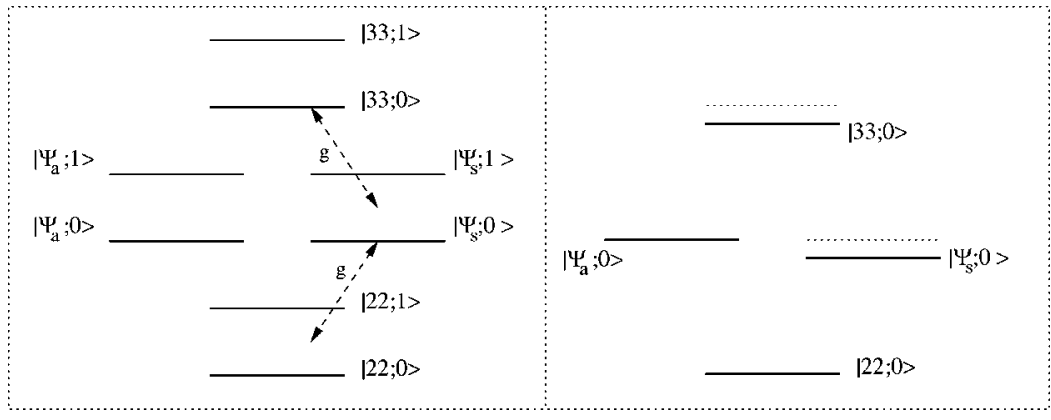

FIG. 3. Selected energy levels of the ions for both zero- and one-photon levels. Due to the detuned atom-cavity interaction (indicated by arrows), energy shifts occur in some of the zerophoton levels $\left[\left|\Psi_{s}\right\rangle=1 / \sqrt{2}(|23\rangle+|32\rangle)\right]$. 


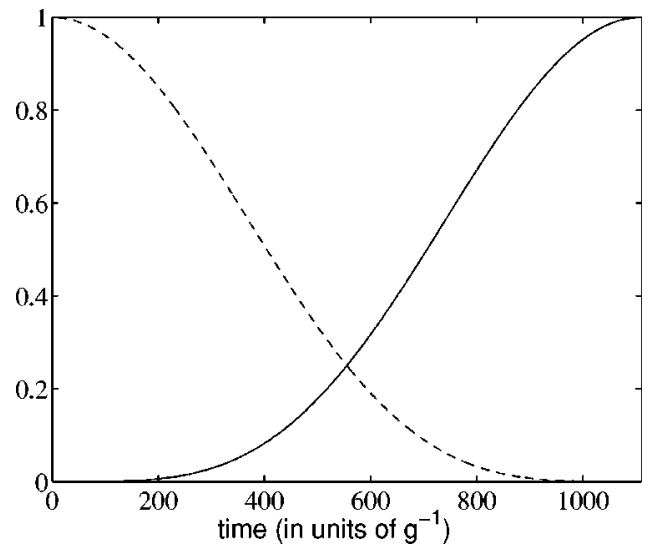

FIG. 4. Oscillations between the states $1 / \sqrt{2}(|11\rangle+|22\rangle)$ and $1 / \sqrt{2}(|11\rangle-|22\rangle)$ when there is no dissipation. In the figure one can see the populations of both states. The parameters are $\Delta=3 g$ and $\Omega=2.0 \times 10^{-3} \mathrm{~g}$. The probability of having zero photons is $>0.999$.

sponding to zero photon states. Choosing a suitably detuned laser, we can therefore selectively couple energy levels. As the energy shifts arise due to the interaction with a cavity mode that is nonlocal, we obtain an effective nonlocal dynamics when shining lasers on individual ions. This idea is reminiscent of the use of free-space dipole-dipole interaction between extremely closely spaced ions [15] but here the interaction is mediated by the cavity mode. It should be noted that in our setup the ions can have a distance of many optical wavelengths at which the free-space dipole-dipole interaction is negligible.

In order to see whether the above approximations are correct, we have studied numerically the system using the full Hamiltonian without any approximations. For instance, we would expect oscillations between the state $1 / \sqrt{2}(|22\rangle$ $+|11\rangle)$ and $1 / \sqrt{2}(|22\rangle-|11\rangle)$. In Fig. 4 we plot the oscillations between these two states for the parameters $\Delta=3.0 \mathrm{~g}$

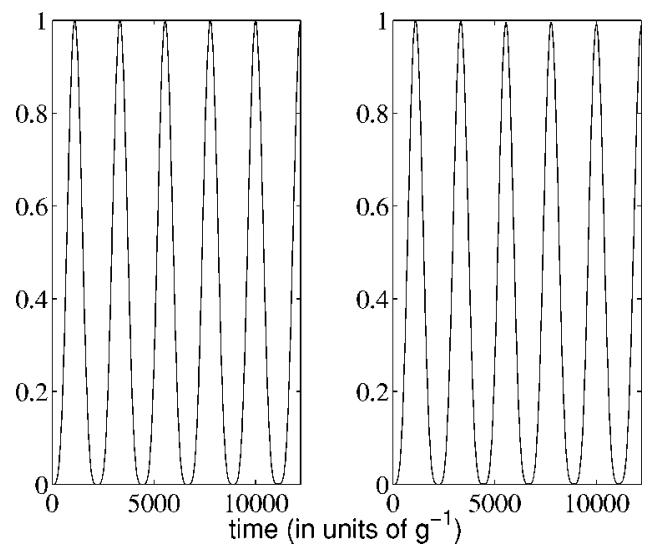

FIG. 5. Fidelity of the gate starting from the state $1 / \sqrt{2}(|11\rangle$ $+|22\rangle$ ) without cavity decay, i.e., $\kappa=0$ (left) and with cavity decay $\kappa=1.0 \mathrm{~g}$ (right). The gate is performed for five consecutive times. The parameters are $\Delta=3.0 \mathrm{~g}$ and $\Omega=2.0 \times 10^{-3} \mathrm{~g}$. Note that this choice of parameters is well within the reach as a cavity with $0.5 \mathrm{~mm}$ length at $866 \mathrm{~nm}$ allows for a ratio of $g / \kappa \approx 27$ [11]. The similarity of the two graphs demonstrates that the influence of the cavity decay is efficiently suppressed.

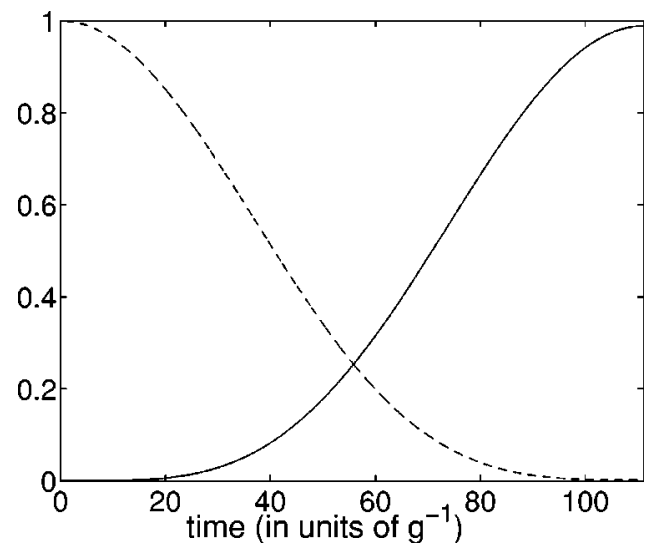

FIG. 6. Oscillations between the states $1 / \sqrt{2}(|11\rangle+|22\rangle)$ and $1 / \sqrt{2}(|11\rangle-|22\rangle)$. In the figure one can see the populations of both states. The parameters are $\Delta=3.0 \mathrm{~g}, \Omega=2.0 \times 10^{-2} \mathrm{~g}$, the cavity decay rate $\kappa=0.5 \mathrm{~g}$, and $\Gamma=2.0 \times 10^{-4} \mathrm{~g}$ for the linewidth of the upper level.

and $\Omega=0.01 g$. We observe that although $(g / \Delta)^{2} \sim 0.1$, the photon population remains vanishingly small for all practical purposes. It is important to note that in this scheme the interaction of the cavity mode with the two ions is the ingredient that allows us to construct the two-qubit gate although the cavity mode has (almost) no population throughout the performance of the gate. This becomes important when the cavity has losses since in principle this will not affect this procedure.

It remains to be seen whether any one-qubit gate can be performed within this setup, since single-qubit operations are required to achieve universal quantum computation. A general single-qubit rotation can be achieved by coupling the two levels $|1\rangle$ and $|2\rangle$ of one ion via a Raman transition by using two lasers with the appropriate phases. This transition can be done using another level that is not coupled to the cavity and does not suffer any energy shift. This framework allows us to work with states with a very long lifetime and therefore the decoherence due to spontaneous emission of the ions when they are in the lower levels is strongly suppressed.

In the analysis so far we have neglected the cavity decay. In a practical experimental situation, however, such process is present and for practical purposes, is important to know whether the scheme is robust against decoherence. Fortunately, it turns out that the use of a far detuned cavity makes the scheme robust against cavity decay as the cavity modes have an extremely small population throughout the gate operation. This is demonstrated in Fig. 5 where we have run a simulation with vanishing $\kappa$ (left half of the graph) and $\kappa$ $=g$ (right half of the graph). There is no appreciable difference between the two time evolutions, which clearly shows that the influence of the cavity decay is efficiently suppressed.

A further source of decoherence in this proposal is the fact that the state $|3\rangle$ can decay spontaneously to $|2\rangle$ since the upper level is populated during the evolution. On the other hand, the gate can be performed fast because even when we have $g \sim \Delta$ there are no photons created in the cavity. In Fig. 
6 we show a Monte Carlo simulation [16] where we see that we have a gate fidelity of $\sim 0.99$ for a decay rate of $\Gamma$ $=5.0 \times 10^{-5} \mathrm{~g}$.

In summary, we have demonstrated that a system consisting of ions trapped inside of a far detuned optical cavity can be used to implement coherent quantum-information processing. The strong detuning between cavity mode and ion leads to an effective suppression of the cavity decay. Spontaneous decay from the ions is reduced by storing the qubits in two lower states of a Raman configuration. Both single qubit and two qubit can be implemented. Since the ions can be moved inside and outside the cavity [12], our proposal can achieve universal quantum computation. The scheme can be implemented with current experimental technology as the recent preparation of trapped ions in optical cavities demonstrates [12].

We would like to thank J. Pachos for discussions and for bringing his closely related results [17] to our attention before publication. This work was partially supported by EPSRC, the European Union EQUIP project the ESF QIT program, and Grant Nos. MEC (AP99), IUAP-P4-02, and GOA-Mefisto-666. This work was partially supported by Grant No. 43371-PH-QC from the U.S. Army Research Office.
[1] M.B. Plenio and V. Vedral, Contemp. Phys. 39, 431 (1998); M. A. Nielsen and I. J. Chuang, Quantum Computation and Quantum-Information (Cambridge University Press, Cambridge, 2000)

[2] D.J. Wineland et al., J. Res. Natl. Inst. Stand. Technol. 103, 259 (1998); C. Monroe et al., Phys. Rev. Lett. 75, 4714 (1995); B.E. King et al., ibid. 81, 1525 (1998); Q.A. Turchette et al., ibid. 81, 3631 (1998); Ch. Roos et al., ibid. 83, 4713 (1999); C.A. Sackett et al., Nature (London) 404, 256 (2000).

[3] J.I. Cirac and P. Zoller, Phys. Rev. Lett. 74, 4091 (1995).

[4] M.B. Plenio and P.L. Knight, Phys. Rev. A 53, 2986 (1996); R.J. Hughes et al., Phys. Rev. Lett. 77, 3240 (1996); M.B. Plenio and P.L. Knight, Proc. R. Soc. London, Ser. A 453, 2017 (1997); A. Steane, et al., Phys. Rev. A 62, 042305 (2000).

[5] A. Sorensen and K. Molmer, Phys. Rev. Lett. 82, 1971 (1999); Fortschr. Phys. 48, 811 (2000).

[6] D. Jonathan and M.B. Plenio, Phys. Rev. Lett. 87, 127901 (2001); D. Jonathan, M.B. Plenio, and P.L. Knight, Phys. Rev. A 62, 042305 (2000).

[7] S. Schneider, D.F.V. James, and G.J. Milburn, J. Mod. Opt. 47, 499 (2000).

[8] G. Morigi, J. Eschner, and C.H. Keitel, Phys. Rev. Lett. 85, 4458 (2000); F. Schmidt-Kaler, J. Eschner, G. Morigi, C.F.
Roos, D. Leibfried, A. Mundt, and R. Blatt, e-print quant-ph/0107087.

[9] M.B. Plenio, S.F. Huelga, A. Beige, and P.L. Knight, Phys. Rev. A 59, 2468 (1999); A. Beige, D. Braun, S. Bose, S.F. Huelga, P.L. Knight, M.B. Plenio, and V. Vedral, J. Mod. Opt. 47, 2583 (2000); A. Beige, D. Braun, and P.L. Knight, New J. Phys. 2, 22 (2000); B. Tregenna, A. Beige, and P.L. Knight, Phys. Rev. A 65, 032305 (2002); A. Beige, D. Braun, B. Tregenna, and P.L. Knight, Phys. Rev. Lett. 85, 1762 (2000).

[10] S. Bose, P.L. Knight, M.B. Plenio, and V. Vedral, Phys. Rev. Lett. 83, 5158 (1999); Pramana, J. Phys. 56, 383 (2001).

[11] W. Lange (private communication).

[12] W. Lange, in Quantum-Information: Theory, Experiment and Perspectives, Second ESF-QIT Programme Conference, Gdansk, 2001 (unpublished); F. Schmidt-Kaler, ibid.

[13] In order to be able to do perturbation theory, the condition $\Omega$ $\gg g^{4} / \Delta^{3}$ has to be satisfied, since Eq. (2) has corrections of order $O\left(g^{4} / \Delta^{4}\right)$.

[14] D.F.V. James, Phys. Rev. Lett. 81, 317 (1998).

[15] A. Beige, S.F. Huelga, P.L. Knight, M.B. Plenio, and R.C. Thompson, J. Mod. Opt. 47, 401 (2000).

[16] M.B. Plenio and P. Knight, Rev. Mod. Phys. 70, 101 (1998), and references therein.

[17] J. Pachos and H. Walther, e-print quant-ph/0111088. 